\title{
Transmisión textual y catálogo de la obra poética de Pedro de Gracia Dei*
}

\author{
Natalia Anaís Mangas NAVArro \\ nataliamangas_4@hotmail.com \\ Universitat d'Alacant
}

La amplia difusión de la obra en verso de Pedro de Gracia Dei nos ha dejado como resultado no solo un considerable número de fuentes, sino también de textos poéticos, con importantes variaciones entre ellos en función del testimonio en que se transmiten. Las composiciones más susceptibles de ser alteradas son las de carácter heráldico y genealógico (ID 1941, ID 6967 bis y Casas y solares nobles de España); basta con advertir las múltiples lecturas y la falta de sistematicidad en las estructuras métricas. El grado de deturpación de estas obras es, probablemente, muy alto en algunos casos, ya que han sido sometidas a versiones o reelaboraciones que las distancian de la que pudo ser la voluntad original del autor. Por el contrario, los textos que se adscriben al género de la historiografía en verso, así como los poemas dedicados a la reina Isabel, presentan una estructura más regular en el conjunto de la tradición textual, con alteraciones de menor calado que, en pocos casos, implican variación en el esquema métrico o en el contenido.

Los títulos con que se transmiten las obras tampoco están exentos de confusión, debido a que se pueden alternar dos o tres rúbricas distintas para un mismo texto, como ocurre, por ejemplo, con los poemas ID 1942 o ID 1943; más inusual, aunque existen casos, es el uso de un título -si no igual, muy parecido- para dos obras diferentes, como encontramos en el ms. 6175 de la BNE. Finalmente, cabe señalar la transmisión fragmentaria de los textos poéticos en algunos testimonios, de manera que para su correcta identificación no es suficiente atender solo a los títulos, íncipits y éxplicits, sino al contenido de cada una de las estrofas, especialmente en aquellas obras de mayor extensión.

Debido a la poca atención que ha recibido la obra poética de Pedro de Gracia Dei, estos complejos procesos de transmisión textual no han sido abordados, con lo que ello implica no solo para la identificación de las

\footnotetext{
* Este trabajo se enmarca en el proyecto Cancionero, romancero y fuentes impresas, del Ministerio de Economía, Industria y Competitividad (FFI2017-86313-P), financiado por la Agencia Estatal de Investigación (AEI) y el Fondo Europeo de Desarrollo Regional (FEDER/ UE), cuyo investigador principal es Josep Lluís Martos.
} 
obras, sino también para su delimitación, ya que la copia fragmentaria de algunas composiciones puede inducir a error. El resultado, en efecto, se evidencia en la ausencia de un corpus poético concreto -y completode Pedro de Gracia Dei, que refleje todas las noticias de que disponemos actualmente. No obstante, varios han sido los intentos de catalogación de su obra, pues ya Gayangos, pese a la antigüedad de su trabajo, esbozó el esquema que más se aproxima a día de hoy ${ }^{1}$. Un siglo después, Dutton nos brindará El cancionero del siglo $\mathrm{XV}$, con datos que serán asumidos $\mathrm{y}$, así, trasladados, a distintos trabajos posteriores ${ }^{2}$.

Sin embargo, las limitaciones que presenta El cancionero del siglo $X V$ con relación a las fuentes poéticas de Gracia Dei condicionan, inevitablemente, la indexación de los textos. Dutton identifica un total de diez obras a partir de cuatro testimonios: MN57, MP2, NH6 y 89*GD. En el caso de los manuscritos, se trata de fuentes de una considerable riqueza textual, pero el estudio de otras 22 fuentes localizadas nos deja como resultado varias composiciones que no transmiten ninguno de los testimonios catalogados por Dutton, de manera que no tienen un ID asignado.

\section{HERÁLDICA Y GENEALOGÍA}

Dentro de la actividad literaria de Pedro de Gracia Dei destacan, notablemente, la heráldica y la genealogía, disciplinas a las que podemos adscribir un total de cuatro obras. El poema titulado Blasones de las armas e insignias de los mejores y más principales linajes de Castilla (ID 1941) es, sin duda, el que presenta más problemas de transmisión en el conjunto de la tradición textual. La diversidad de estrofas que convergen en cada una de las fuentes nos impide establecer una estructura métrica fija, así como unos íncipits o éxplicits concretos. Dutton identifica esta obra en el cancionero NH6, si bien se transmite en un total de catorce testimonios más, aunque con notables diferencias en el esquema métrico. Con el mismo título que en NH6, recogen el poema los mss. 3231,

\footnotetext{
${ }^{1}$ Pedro de Gracia Dei, Blasón General y Nobleza del Universo, prólogo de Pascual de Gayangos, Madrid, Imprenta M. Murillo, 1882, ed. facsímil, Badajoz, Unión de Bibliófilos Extremeños, 1993, pp. XI-XV.

${ }^{2}$ Víctor Infantes, «La Cortesía en verso de Pedro de Gracia Dei y su tratado La Criança y virtuosa doctrina (1488)», en Rose Duroux (ed.), Traités de savoir-vivre en Espagne et au Portugal du Moyen Âge à nos jours, Clermont Ferrand, Association des Publications de la Faculté des Lettres et Sciences Humanines de Clermont Ferrand, 1995, pp. 45-48; Óscar Perea Rodríguez, «"Alta reina esclarecida”: un cancionero ficticio para Isabel la Católica», en Luis Antonio Ribot García, Julio Valdeón Baruque y Elena Maza Zorrilla (coords.), Isabel la Católica y su época: actas del Congreso Internacional. Valladolid-Barcelona-Granada (15-20 noviembre, 2004), Valladolid, Universidad de Valladolid, 2007, vol. 2, pp. 1355-1383; JeanPierre Jardin, «Pedro de Gracia Dei, roi d'armes et poète. Entre historie, littérature et arts graphiques», e-Spania, 23 (2016). En línea. DOI: https://doi.org/10.4000/e-spania.25239; Ruth Martínez Alcorlo, La literatura en torno a la primogénita de los Reyes Católicos: Isabel de Castilla y Aragón, princesa y reina de Portugal (1470-1498), [Tesis Doctoral], Madrid, Universidad de Complutense de Madrid, 2016.
} 
3449, 3769, 5911, 7864 y 18045 de la BNE, el ms. 69 de la Biblioteca Lambert Mata, el ms. 58-4-6 de la Biblioteca Capitular de Sevilla y el COD. 1155 de la Biblioteca Nacional de Portugal. El ms. 6175 de la BNE incluye el texto bajo la rúbrica Linages y blasones y en el ms. 3564 de la BNE se introduce sin título. Finalmente, el cancionero MP2, los mss. 1367, 12612 de la BNE, II/660 de la Real Biblioteca y 50-I-41 de la Biblioteca da Ajuda transmiten algunas estrofas de ID 1941, pero se alternan con coplas que forman parte de los Blasones de ciudades y villas (ID $6967^{\text {bis }}$ ).

A pesar de las importantes variaciones entre unas fuentes y otras, sí podemos advertir, en cambio, un orden en la transmisión de los blasones que, hasta cierto punto, podríamos caracterizar de sistemático. La mayoría de los manuscritos presentan la siguiente secuencia de apellidos: Manrique, Lara, conde de Osorno, marqués de Aguilar, Ponces de León, Haro, Guzmán, Guzmanes de Sevilla, Enríquez, Manueles, de la Cerda, los de Castilla, Mendozas, Laso de la Vega, Castros, duque de Villahermosa, Estúñiga, Velasco, Guevara, Rojas, Toledo, Ávila, Córdoba, Meneses, Pimentel, Portugal, Silva, Puerto Carrero, Coello, Fonseca, Pachecos, Acuña, Bocanegra, Girón, Téllez y Molina, Sarmiento, Arellanos, Osorios, Quiñones, Carrillo, Cárdenas, Tovar, Salazar, Luna, Villandrando, de la Cueva, Ávalos, Padilla, Herrera, Coronel, Sotomayor, Figueroa, Maldonado, Arce, Cabeza de Vaca, Reinoso, Cisneros, Solier, Barba, Biedma, Leiva, Lucena, Loaysa, Montoya, de la Torre, Peralta, los de Paraje, Espinosa de los Monteros, Cartagena, Saldaña y Guirana.

Hasta el apellido de Guirana, esta secuencia se transmite igual en el cancionero NH6, los mss. 3231, 3449, 3769, 5911 y 7864 de la BNE, el ms. 58-4-6 de la Biblioteca Capitular de Sevilla y el COD. 1155 de la Biblioteca Nacional de Portugal. A partir de ese linaje, cada fuente continúa con un orden distinto, si bien es idéntico en cada una de las familias de manuscritos que podemos identificar: en NH6 y en los mss. 3231, 3769 y 5911 de la BNE; por otra parte, en los mss. 7864 de la BNE, el ms. 58-4-6 de la Biblioteca Capitular de Sevilla y el COD. 1155 de la Biblioteca Nacional de Portugal; finalmente, en los mss. 18045 de la BNE y el ms. 69 de la Biblioteca Lambert Mata.

La complejidad de esta obra se evidencia, en última instancia, por la duplicación del ID 1941 en El cancionero del siglo XV: Dutton asigna el mismo ID a una de las composiciones que contiene MP2, titulada Coplas de Gracia Dey en loor de algunos linajes y ciudades de Castilla. Toro Pascua, basándose en el cancionero NH6 y en el ms. 69 de la Biblioteca Lambert Mata, intenta solventar este problema de identificación: considera que la confusión de Dutton reside en la coincidencia de una de las coplas entre los tres testimonios; concretamente, la primera estrofa de los Blasones de las armas e insignias en NH6 y en el ms. 69 
de Biblioteca Lambert Mata es la misma que la tercera en el texto que transmite MP2 ${ }^{3}$.

Estos datos, sin embargo, no son correctos. Por un lado, la primera estrofa en NH6 y en el ms. 69 de la Biblioteca Lambert Mata es la que se corresponde con el linaje de los Manrique -«Con parte de las reales / en purpura vi dos pieças»- que, en MP2, ocupa el cuarto lugar y no el tercero; por otro lado, no solo existen coincidencias en la transmisión de esa copla, sino que el conjunto de estrofas que recoge MP2 procede de dos obras distintas: los Blasones de las armas e insignias... (ID 1941 a partir de NH6) y los Blasones de ciudades y villas (ID 6967 ${ }^{\mathrm{bi}}$ ). De hecho, el título que figura en MP2 así lo sugiere: Coplas en loor de linajes y ciudades de Castilla. La estructura que presenta MP2 se transmite de manera idéntica en el ms. 1367 de la BNE, bajo el título Descendencia de algunos linages nobles de castilla compuestos en metro por Garcia del [sic $]^{4}$.

De estos datos inferimos, por tanto, que Dutton asignó un mismo ID a dos textos que consideró iguales en contenido, pero con distinto orden de estrofas. No obstante, sí parece probable que realizase esta identificación a partir de los primeros versos de NH6 y MP2, de manera que no advirtió en este último estrofas procedentes de una obra distinta (ID $6967^{\text {bis }}$ ). El hecho de encontrar dos poemas que comparten ID nos lleva a pensar que se trata de la misma composición; sin embargo, como prueba la estructura métrica y el orden de los blasones, nos encontramos ante un texto distinto. En cualquier caso, el resultado parece responder a un particular proceso de copia, en que el compilador, como demuestra el contenido, tiene acceso a varias obras de Pedro de Gracia Dei y yuxtapone distintas estrofas al azar. Este proceso, por tanto, es ajeno a la voluntad del autor, de manera que no resultaría lícito otorgar a Gracia Dei la autoría de esta composición.

Es por ello que, en este caso, mantenemos el ID 1941 para los Blasones de las armas e insignias de los principales linajes de Castilla, tal y como se transmite en el cancionero NH6 y en otros muchos testimonios, por ser una obra dedicada exclusivamente a blasones nobiliarios $\mathrm{y}$, probablemente, por ser la que presente más cercanía con la que pudo componer Pedro de Gracia Dei. En esta obra, el autor no entra a la descripción de emblemas de ciudades o villas, pues es una cuestión que ya tendrá eco en otro texto. Para el texto que recogen MP2 y el ms. 1367 de la BNE, sin embargo, habría que consignar un número de identificación

${ }^{3}$ María Isabel Toro Pascua, «Nuevos y viejos poemas para el "Cancionero del siglo Xv (c. 1360-1520": fuentes manuscritas», en Javier San José Lera (coord.), Praestans labore Victor: homenaje al profesor Víctor Garcia de la Concha, Salamanca, Universidad de Salamanca, 2005, p. 85.

${ }^{4}$ La única diferencia entre un manuscrito y otro reside en que el ms. 1367 de la BNE incluye una estrofa más, dedicada a los Ramírez, situada entre los Osorios y los Bravos. Respecto a la estructura métrica, en esta fuente los linajes de Puerto Carrero y Andradas tienen diez versos, mientras que en MP2 las estrofas dedicadas a esos apellidos están formadas por eneasílabos. 
distinto, como, por ejemplo, ID $1941^{\text {bis }}$ o ID $6967^{\text {bis }}$ o, simplemente, señalar que nos encontramos ante una composición formada por estrofas que provienen de dos obras diferentes.

Tampoco está exento de confusión el poema titulado Blasones de ciudades y villas (ID 6967 $7^{\text {bis }}$ ) que, en la línea de ID 1941, se transmite de forma muy variopinta en el conjunto de los testimonios. La versión más completa que nos ha llegado de este texto es la que recogen NH6 y los mss. 3231, 3769 y 5911 de la BNE, formada por 28 estrofas. En los mss. 7864 de la BNE, 58-4-6 de la Biblioteca Capitular de Sevilla y el COD. 1155 de la Biblioteca Nacional de Portugal la obra contiene 23 estrofas. El resto de fuentes que transmiten el poema incluyen distintas coplas que no se atienen a un orden sistemático, ni tampoco al que presentan los manuscritos anteriores. Es el caso de los mss. 3449, 6175 y 12612 de la BNE, el ms. II/660 de la Real Biblioteca y el ms. 50-I-41 de la Biblioteca da Ajuda. El ms. 1367 de la BNE y MP2, como ya hemos advertido a propósito de ID 1941, contienen también varias estrofas que forman parte de esta obra. No obstante, en lo que respecta a la primera copla, existe cierta regularidad, ya que la mayoría de las fuentes comienzan con la estrofa dedicada a la villa de Carrión ( «Vi el carro triumphal / de la noble Carrión») o bien por Castilla («Para blasonar a Castilla / es tan alta su substancia»).

Dentro de las disciplinas de la heráldica y la genealogía, debemos incluir dos obras de Pedro de Gracia Dei que no documenta Dutton, de manera que no tienen un ID asignado. Uno de ellas se titula La universal: Coplas de los escudos de los principes del mundo, incluida en seis testimonios, todos ellos de la BNE: los mss. 3231, 3769, 5911, 9087, 18053 y 18380 . El contenido de cada una de las estrofas es muy similar al que presenta una parte del Libro de armería de Hernández de Mendoza, donde se describen los siguientes emblemas: las armas de la ciudad de Jerusalén y de Sicilia; de los reinos de Portugal, Inglaterra, Escocia, Noruega, Hibernia, Bohemia, Hungría, Frisa, Nápoles; el origen y armas de la ciudad de Francia; armas de los emperadores de Alemania, Grecia y del duque de Milán; armas de los reyes de Castilla, Navarra, Aragón, Panonia y León, Suecia y Gocia y Chipre; finalmente, las armas del gran maestre de San Juan y del preste Juan de las Indias. Es por ello que, en esta línea, en los mss. 9087, 18053 y 18380 se combinan las coplas de Pedro de Gracia Dei con la parte en prosa del Libro de armería en que se describen esos blasones.

La obra está formada por 24 estrofas, ya que así nos ha llegado a través de las fuentes que la incluyen, a excepción del ms. 18380, que recoge un total de 17 coplas. Gayangos ya dio cuenta de esta obra, aunque erróneamente sostiene que «es la misma ya citada bajo el núm. II con 
el título de Genealogía de los Reyes de España en coplas» ${ }^{5}$ (1882: xv) que, según el íncipit que anota, se trataría de otra composición de Pedro de Gracia Dei, concretamente la que Dutton identifica con el ID 6966 $6^{\text {bis }}$ (De los Reyes Godos).

El otro texto de carácter heráldico que tampoco recoge El cancionero del siglo $X V$ se transmite únicamente en los siguientes testimonios: los mss. 3231, 3769, 5911 y 18053 de la BNE. Como se puede advertir, se trata, en los cuatro casos, de los manuscritos que también incluyen las Coplas de los escudos de los príncipes del mundo; de hecho, se copia siempre a continuación de esa obra. El título con que se identifica el texto resulta, todavía a día de hoy, confuso. En los mss. 3231, 3769 y 5911 no figura ninguna rúbrica, sino que, tras la última estrofa de las Coplas de los escudos de los príncipes del mundo, que se corresponde con las armas del preste Juan de las Indias, se indica que «Comienzan los escudos en A. De las armas de los Arellanos», anotación que se repite de manera sistemática para todos los linajes, ordenados alfabéticamente. Solo el ms. 18053 intitula la obra como Comiençan las armas y blasones de los cavalleros y nobles despaña. No obstante, tanto el IGM como BETA identifican el texto con el título La Universal: Casas y solares nobles de España.

La versión más extensa de la obra es la que transmite el ms. 18053, formada por 285 estrofas, aunque, al igual que ocurre en algunas fuentes que transmiten las Coplas de los escudos de los príncipes del mundo, se alternan con partes en prosa que forman parte del Libro de armería. En este punto, resulta complicado advertir si realmente todas las coplas incluidas en esta obra son de la pluma de Gracia Dei, máxime si tenemos en cuenta las múltiples alteraciones que sufren los textos de esta naturaleza; no obstante, Gayangos sí considera este Nobiliario, pues así se titula el manuscrito, como original de Gracia Dei ${ }^{6}$. Tampoco podemos cotejar el texto que transmite el ms. 18053 con los otros testimonios, puesto que presenta unos contenidos y esquema métrico diferentes: la obra se interrumpe con el apellido Esquiveles en los mss. 3231, 3769 y 5911 de la BNE («con sus tiempos no noveles / matiz de sus blasones») y contiene un total de 111 estrofas, en contraste con el ms. 18053, que llega hasta el linaje de los Sarmiento.

\section{HISTORIOGRAFÍA}

Otro de los campos predilectos de Pedro de Gracia Dei es la historiografía, que aborda desde una perspectiva claramente neogoticista: los monarcas castellanos se presentan como herederos directos de los

\footnotetext{
${ }^{5}$ Pedro de Gracia Dei, op. cit., p. Xv.

${ }^{6}$ Pedro de Gracia Dei, op. cit., p. IX.
} 
reyes visigodos. Así, las dos obras que podemos englobar dentro de este género se atienen a un marco temático muy concreto y el desarrollo de los contenidos se lleva a cabo de forma similar: una primera parte está dedicada a todos los reyes godos, desde Atanarico hasta Rodrigo, mientras que la segunda parte comienza con don Pelayo y culmina con los Reyes Católicos. Se establece, así, una línea ininterrumpida entre los monarcas visigodos y los reyes castellanos, aragoneses y astur-leoneses. Advertimos, pues, un salto histórico-temporal de unos pocos años, ya que el autor omite intencionadamente la irrupción musulmana en la Península en el 711, año en que fue destituido Rodrigo, el último rey godo, y retoma el hilo de la historia con don Pelayo, como figura clave que inicia el período de la Reconquista. De hecho, esta segunda parte suele ir encabezada por la rúbrica «Restauración de España», al mismo tiempo que se identifica a don Pelayo como el «restaurante».

La obra más extensa dentro de este género es la que Dutton identifica en NH6 como De los Reyes Godos (ID 6966 ${ }^{\text {bis }}$ ), aunque, en realidad, ese título se corresponde con una rúbrica interestrófica, como otras tantas que presenta el texto; quizá, ni siquiera formaran parte de la composición que concibió originariamente Gracia Dei y sea resultado de los múltiples procesos de copia. Esta obra siempre va acompañada de una copla real, que realiza la función de proemio y que Dutton documenta como Genealogía y blasón de los Reyes de Castilla (ID 6965 ${ }^{\text {bis }}$ ). Este título, sin embargo, es el que se correspondería con el conjunto de la obra y no solo con el proemio, debido a la relación evidente entre rúbrica y contenido. De hecho, De los Reyes Godos solo hace alusión a un número determinado de estrofas, ya que el texto también incluye a los monarcas castellanos, aragoneses y astur-leoneses. Quizá, resultaría más adecuado, además de aclaratorio, englobar bajo el título Genealogía y blasón de los Reyes de Castilla el texto completo, incluyendo el proemio, como ya identificó Gayangos ${ }^{7}$ y como, actualmente, consignan el IGM y BETA, más allá de que en las descripciones internas se indique la existencia de una copla real como encabezamiento del poema.

En cualquier caso, el ID del proemio (ID $6965^{\text {bis }}$ ) no se corresponde con la metodología habitual que sigue Dutton cuando identifica un texto como introducción de otro, para lo que utiliza la letra «I» entre dos IDs. Este aspecto nos llevaría a modificaciones en el número de identificación, sugiriendo el ID $6965^{\text {bis }}$ I $6966^{\text {bis }}$, como ya advirtió Toro Pascua ${ }^{8}$. Como es natural, todos los testimonios que transmiten la obra De los Reyes Godos (ID 6966 ${ }^{\text {bis }}$, en la línea de Dutton) incluyen, también, el proemio (ID 6965 ${ }^{\text {bis }}$ ): se trata del cancionero NH6, los mss. 3231, 3449, 3564, 7864 y 18045 de la BNE, el ms. 69 de la Biblioteca Lambert Mata,

\footnotetext{
${ }^{7}$ Pedro de Gracia Dei, op. cit., p. XI.

${ }^{8}$ María Isabel Toro Pascua, art. cit., p. 86.
} 
el ms. 58-4-6 de la Biblioteca Capitular de Sevilla, el COD. 1155 de la Biblioteca Nacional de Portugal y el ms. 50-I-41 de la Biblioteca da Ajuda. En la mayoría de los testimonios, la obra está compuesta por 87 estrofas, aunque el ms. 3231 de la BNE transmite 81 y el ms. 50-I-41 de la Biblioteca Da Ajuda incluye un total de 75 .

Al margen de las confusiones que implican los números de identificación asignados por Dutton, el principal problema que presenta este texto reside en el título con que se transmite en los mss. 3769 y 5911 de la BNE: Vergel de nobles dedicado al Gran Capitán. Este aspecto ha llevado a consignar ese Vergel de nobles como una obra independiente de Pedro de Gracia Dei ${ }^{9}$ cuando, en realidad, se trata del poema ID $6966^{\text {bis }}$, pero copiado de manera fragmentaria: no comienza desde el rey Atanarico, como en el resto de fuentes, sino desde el rey Sisnando, que ocupa la estrofa $\mathrm{n}^{\circ} 23$ del texto completo, es decir, del que nos ha llegado formado por 87 coplas. Esto implica que, además de que no incluyen el proemio (ID 6965 bis), el íncipit es diferente y, quizá, ha sido ese aspecto el que ha despistado a la crítica.

Es importante señalar también una característica que se ha pasado por alto y que condiciona la estructura métrica de la obra: la estrofa que se corresponde con el reinado de Juan I contiene nueve versos. Si atendemos al esquema métrico de la copla real (abaabcdccd) faltaría el cuarto, que, en algunos manuscritos, el copista evidencia con un espacio en blanco entre el tercer y el quinto verso. La falta se repite en todos los testimonios que transmiten el texto, a excepción del COD. 1155 de la Biblioteca Nacional de Portugal, el único que propone una lectura para el cuarto verso: «acordar a su tributo». Dutton no especifica la estructura métrica de esta obra en la descripción que realiza de NH6, pero sí lo hace Toro Pascua en relación al manuscrito 69 de la Biblioteca Lambert Mata, aunque indica, erróneamente $« 87 \times 10 »^{10}$.

Mientras que De los Reyes Godos se caracteriza por dedicar una estrofa a cada uno de los monarcas - de ahí su considerable extensión-, en el Tratado de todos los reyes que en España ha habido desde los godos se incluyen dos e, incluso, tres reyes por copla, si bien el recorrido histórico es el mismo: desde Atanarico hasta los Reyes Católicos. Dutton asigna el ID 1942 a partir de una obra que recogen MP2 y NH6 e indica el título La descendencia de los Reyes de Castilla. No obstante, según su identificación, existen diferencias entre ambos textos, ya que MP2

${ }^{9}$ Víctor Infantes, art. cit., p. 46; Óscar Perea Rodríguez, «"Alta reina esclarecida”..., art. cit., p. 1373; Martínez Alcorlo, op. cit., p. 218. En efecto, Infantes indica que los mss. 3231, 3769 y 5911 de la BNE recogen una obra titulada Vergel de Nobles, que se corresponde con lo que aquí identifico como un fragmento de la Genealogía y Blasón de los Reyes de Castilla -o de los Reyes Godos, siguiendo el título de Dutton. Martínez Alcorlo, además, indica la existencia de una copia de ese «Vergel de Nobles» en la BNE, con signatura 9133. No queda rastro de ninguna obra de Gracia Dei en ese testimonio, que únicamente incluye cartas de Diego de la Fuente.

${ }^{10}$ María Isabel Toro Pascua, art. cit., p. 85. 
contiene una secuencia de tres estrofas al final de ID 1942 que solo incluye este testimonio y que Dutton consigna como parte de este poema ${ }^{11}$. En realidad, nos encontramos ante otro peculiar proceso de copia, ya que la línea temática que siguen estas estrofas se distancia de la que presenta el Tratado de todos los reyes que en España ha habido desde los godos.

La estructura que recoge NH6, formada por 19 décimas, es la que se repite en todas las fuentes que transmiten la obra, independientemente de la filiación que exista entre ellas: los mss. 3231, 3449, 3564, 7864 y 18045 de a BNE, el ms. 58-4-6 de la Biblioteca Capitular de Sevilla, el ms. 69 de la Biblioteca Lambert Mata, el códice 50-I-41 de la Biblioteca da Ayuda y el COD. 1155 de la Biblioteca Nacional de Portugal. Esto nos lleva, por tanto, a considerar esa estructura como la más cercana a la voluntad del autor. La particularidad de la obra reside en la última estrofa, que se corresponde con la tercera copla de otro texto de Gracia Dei, Las quinze preguntas que hizo el Papa Julio a Gracia Dei sobre las excelencias de la reina doña Ysabel, dirigido a Isabel la Católica: «Qué hizo». Ínc.: «Vençio treze reyes cristianos / recobro sus señorios»; éxpl.: «y nuevas gentes hallando / a Napoles recobro». Puesto que en ID 1942 la última estrofa se dirige conjuntamente a los Reyes Católicos, se toma como modelo esa copla y se conjugan los verbos en plural: «Qué hicieron». Ínc.: «Vencieron treze reyes cristianos / recobraron sus señoríos»; éxpl.: «y nuevas gentes hallando / a Napoles recobraron».

Este proceso de transmisión textual puede tener dos explicaciones: por un lado, que el propio Gracia Dei aprovechase intencionadamente esa estrofa para dos composiciones diferentes. Si tenemos en cuenta que Las quinze preguntas que hizo el Papa Julio... se compusieron a partir del fallecimiento de la reina Isabel, es posterior, por tanto, al Tratado de todos los reyes que en España ha habido desde los godos, de manera que, inicialmente, esa estrofa formaría parte del poema ID 1942. Sin embargo, también cabe la posibilidad de que se trate de una reutilización posterior, ajena al proceso de composición de Gracia Dei y fruto de las compilaciones de los copistas, que conocían ambas obras.

Existen cinco testimonios que no contienen esta última estrofa de ID 1942, de manera que presentan un éxplicit distinto: "son Fernando e Ysabel / los dos bienaventurados». Se trata de los mss. 6175 de la BNE, II/660 de la Real Biblioteca, h. II. 21. del Real Monasterio de El Escorial, F/30 del Monasterio de las Descalzas Reales y 9/271 de la Real Academia de la Historia. A excepción del primero, las otras cuatro fuentes se caracterizan, además, por transmitir el poema ID 1942 de forma fragmentaria, con un total de siete décimas: comienzan desde don Pelayo - estrofa n. ${ }^{\circ} 12$ del texto completo-y no desde el rey Atanarico.

${ }^{11}$ Brian Dutton, El cancionero del siglo XV (c. 1360-1520), Salamanca, Universidad de Salamanca, 1990, tomo II, p. 424. 
Dutton, a partir de MN57, identifica una versión de ID 1942, a la que asigna el ID 8324 V 1942: Suma de todos los reyes que ha habido en España desde los godos. Aunque también está formada por 19 décimas, la principal diferencia entre ambos textos reside de, nuevo, en la última estrofa: MN57 incluye unos versos dedicados a Juana de Castilla y Felipe de Habsburgo que no encontramos en ningún otro testimonio ${ }^{12}$. No obstante, la autoría de esta copla es muy cuestionable, máxime si atendemos a la anotación marginal que la acompaña: «No la hizo Gracia dey».

\section{PAnegíricos a IsABel la CATólica}

Generalmente, al poema ID 1942 le precede una décima (abaabccddc) dirigida a la reina Isabel, que Dutton identifica con ID $6964^{\text {bis }}$ I 1942: Comiença y dirige la obra a la reina doña Isabel, a excepción de MP2 y los mss. 1367 y 6175 de la BNE, que comienzan sin preámbulo con el Tratado de todos los reyes que en España ha habido desde los godos. Esta estrofa, por tanto, se trasmite en el cancionero NH6, en los mss. 3231, 3449, 3564, 7864 y 18045 de la BNE, el ms. 69 de la Biblioteca Lambert Mata, el ms. 58-4-6 de la Biblioteca Capitular de Sevilla, el COD. 1155 de la Biblioteca Nacional de Portugal y el ms. 50-I-41 de la Biblioteca da Ajuda. La primera quintilla de esta décima se corresponde con los cinco primeros versos de la segunda estrofa de Las XV preguntas que hizo el Papa Julio...:
Muy alta muy poderosa
del mundo mayor señora
muy justa, muy piadosa
muy liberal, muy hermosa
y muy recta regidora.

La obra más extensa que nos ha llegado como panegírico a la reina Isabel es, precisamente, Las quinze preguntas que fizo papa Jullio hizo a gracia Dei sobre las excelencias de la Reyna doña Isabel, título por el que se conoce comúnmente a partir de la edición de Paz y Mélia ${ }^{13}$. Esta obra había sido considerada de tradición mono-testimonial, ya que solo teníamos constancia a través del ms. 3346 de la BNE, del que se sirvió Paz y Mélia para su edición. Sin embargo, en una investigación reciente ofrezco una nueva fuente en que se transmite esta composición ${ }^{14}$, el ms.

${ }^{12}$ Cfr. Natalia Anaís Mangas Navarro, «Una fuente de poesía de cancionero: estudio codicológico de MN57», en Josep Lluís Martos y Natalia A. Mangas (eds.), Pragmática y metodologías para el estudio de la poesía medieval, Alacant, Universitat d' Alacant, pp. 161-163.

${ }^{13}$ Antonio Paz y Meliá, Opúsculos literarios de los siglos XIV a XVI, Madrid, Sociedad de Bibliófilos Españoles, 1892, pp. 373-378.

${ }^{14}$ Natalia Anaís Mangas Navarro, «Nuevas fuentes para la poesía de Pedro de Gracia Dei», Revista de Cancioneros Impresos y Manuscritos, 9 (2020), pp. 48 : 54. 
II/660 de la Real Biblioteca, que, asimismo, presenta una rúbrica con algunas variantes con respecto al ms. 3346 de la BNE: Pregvntas Que hiço el papa Julio A graçia dei en las exçelençias de la Reyna doña Ysavel ${ }^{15}$. Además de la rúbrica, advertimos variantes entre los dos testimonios a lo largo de todo el texto, lo que parece indicar que no existe ninguna relación entre ellos, más aun si tenemos en cuenta que el ms. II/660 de la Real Biblioteca de Palacio es, su mayoría, un codex descripti del ms. 12612 de la BNE, mientras el ms. 3346 de la BNE no presenta ningún tipo de filiación con los otros testimonios. Esto nos lleva, por tanto, a presuponer la difusión de la obra a través de distintas fuentes, hoy perdidas o no localizadas, debido a que los copistas de los dos manuscritos debieron de utilizar antígrafos distintos.

Las XV preguntas... es la única composición de Pedro de Gracia Dei que se adscribe al género de las preguntas y respuestas y, a partir del contenido, inferimos que se concibió a raíz del fallecimiento de Isabel la Católica, por lo que podemos establecer como fecha de composición el año 1504; además, en ese año ya era papa Julio II. La obra está formada por 12 quintillas dobles: la primera se corresponde con la pregunta y el resto de estrofas con las respuestas, encabezadas por distintas rúbricas: «Quién era» $(1 \times 10)$, «Qué hizo» $(1 \times 10)$, «Qué ordenó» $(1 \times 10)$, «De qué se honró» $(2 \times 10)$, «Qué tuvo» $(1 \times 10)$, «Qué mandó» (1x10), «Cómo amó al rey» (1x10), «Cómo vivió» (1x10), «Cómo falleció» (1x10) y «Cuánto vivió, cuánto reinó y cuándo y cómo ydo se sepultó» (1x10).

El título de este texto apunta hacia una dirección muy sugerente, que podría aportar importantes datos para la obra poética de Gracia Dei. Gayangos identifica una obra titulada Excelencias de la Christianisima Reyna de España Doña Isabel, dirigida a Juana de Castilla y Felipe de Habsburgo y para la que anota el mismo íncipit que presenta el poema ID 6964 ${ }^{\text {bis }}$ I 1942 $2^{16}$; a su vez, como ya advertimos, ese íncipit se corresponde también con el de la segunda estrofa de Las XV preguntas, pues se trata de la misma quintilla: «Muy alta muy poderosa». La rúbrica del texto que consigna Gayangos es prácticamente idéntica a la de otra obra de Pedro de Gracia Dei hoy perdida, pero que sabemos de su existencia gracias al Regestrum de Hernando Colón: las Exçelencias de la Reina doña Isabel de Castilla en coplas castellanas. Desconocemos, por tanto, su extensión y contenido, pero es probable que esta composición contuviese varias estrofas de Las XV preguntas, si atendemos, especialmente, al título que figura en el manuscrito de la Real Biblioteca: Pregvntas

${ }^{15}$ Perea Rodríguez indica que esta obra se transmite también en NH6 («"Alta reina esclarecida":.., art. cit., p. 1374). La confusión, como suele ser habitual, ha debido de darse por la coincidencia entre la primera quintilla de la segunda estrofa de Las XV preguntas ... y los cinco primeros versos de ID $6964^{\text {bis }}$ I 1942 , texto con el que comienza NH6.

${ }^{16}$ Gracia Dei, op. cit., p. IX. 
que hiço el papa Julio A graçia dei en las exçelençias de la Reyna doña Ysavel. Quizá, «en las excelencias» pueda referirse a esa obra perdida.

Esta hipótesis podría respaldarse si tenemos en cuenta, además, las rúbricas que presentan los mss. 3449 y 7864 de la BNE, el ms. 58-4-6 de la Biblioteca Capitular de Sevilla y el COD. 1155 de la Biblioteca Nacional de Portugal, pues indican que el manuscrito se abre con un texto «lo qual fue sacado de la obra que el dicho Gracia Dei compuso y dirigio a los Reyes Don Felipe y $\mathrm{D}^{\mathrm{a}}$ Joana, de las excelencias de las Christianissimas Reyna $\mathrm{D}^{\mathrm{a}}$ Isavel de gloriosa memoria». La rúbrica coincide con los datos que ofrece Gayangos, tanto el título como los dedicatarios. El poema con que empiezan estos manuscritos es, casualmente, ID 6964 ${ }^{\text {bis }}$ I 1942: «Muy alta muy poderosa / del mundo mayor señora...».

Por tanto, tenemos una quintilla que se transmite en tres textos diferentes: en el poema ID 6964 bis I 1942, en Las XV preguntas y, presumiblemente, en unas Excelencias que, desgraciadamente, no se han conservado. Esta frecuente repetición quizá sí que pueda relacionarse con una reutilización del material poético por parte de Pedro de Gracia Dei, de manera totalmente intencionada: el autor se sirve de una estructura fija para dirigirse a Isabel la Católica y la extrapola de unas obras a otras. No obstante, esto es solo una hipótesis a partir de la relación de los distintos datos.

Dentro de los textos en torno a la reina Isabel, merece especial interés el poema ID 1943, que Dutton identifica a partir de MP2, MN57 y NH6. En los tres testimonios se transmite con títulos diferentes, aunque muy parecidos: Las reynas propietarias (MN57), Esparsa suya en que pone muchos nombres de reinas de españa y Las mugeres reynas en Hespaña (NH6). De hecho, serán las rúbricas que se alternen, indistintamente, en la mayoría de las fuentes, si bien podemos encontrar también el título de Herederas de España. El poema es una décima donde el autor hace referencia a varias reinas e infantas castellano-leonesas de los siglos XII y xIII: Constanza de Borgoña, esposa de Alfonso VI de León; su hija, Urraca I de León; Elvira, infanta de León y reina de Sicilia; y Berenguela de Castilla. Gracia Dei asocia una sola cualidad a cada una de las reinas, mientras que Isabel la Católica, figura protagonista de los dos últimos versos, reúne en su persona todas las características anteriores: «Isabel en cada cosa / perfección de todas ellas». El primer verso de la obra está sujeto a distintas lecturas: aunque la más frecuente se corresponde con «Yberia nombre le dio», en otros manuscritos se transmite «Eugenia»y, en menor medida, «Higeria».

En la descripción interna que Dutton realiza de NH6 le asigna el ID $1943 Y_{6966^{\text {bis }}}$, debido a que aparece seguidamente tras ese texto. Se establece, así, una confusión, ya que le atribuye a ID $6966^{\text {bis }}$ un éxplicit erróneo - «Ysabel en cada cosa / perfection de todas ellas». No obstante, en el índice de autores sí le asigna ya el ID 1943, la identificación 
correcta, pues nos encontramos ante una composición independiente que no se corresponde con el final de ID $6966^{\text {bis }}$. De hecho, no siempre aparece en los testimonios después de ID $6966^{\text {bis }}$, ya que en algunos se copia, también, tras la composición ID 1942. Se trata del texto que se transmite con más frecuencia, ya que nos ha llegado a través de 19 fuentes: todas menos los mss. 3346, 6175, 9087, 18053, 18380 de la BNE y el ms. 50-I-41 de la Biblioteca da Ajuda. Incluso, en algunos manuscritos, el poema se copia dos veces, como ocurre en los mss. 18045 de la BNE y II/660 de la Real Biblioteca.

\section{Obras dedicadas a las hiJas de los Reyes Católicos}

Isabel y Juana también fueron dedicatarias de varias composiciones de Pedro de Gracia Dei. La obra más extensa dentro de este grupo es la Criança y virtuosa dotrina (ID 4692), dirigida a la infanta Isabel, primogénita de los Reyes Católicos. Nos ha llegado a través de un incunable que se erige como el único testimonio impreso poético del autor que se conserva actualmente; además, solo tenemos constancia de un ejemplar, custodiado en la BNE (INC/1272), para el que Dutton asigna la sigla $89 * \mathrm{GD}$. El texto, que consta de 158 coplas de arte mayor, está incompleto, aunque por los datos que nos ofrece el propio autor podemos inferir que, en su origen, estaría formado por 250 estrofas. Nos encontramos ante una peculiar obra en la que confluyen temas de distinta naturaleza, que se desligan por completo de los géneros y disciplinas habituales que cultiva Pedro de Gracia Dei: desde las normas de comportamiento que deben seguir todos aquellos que se encuentran al servicio de reyes y nobles hasta una descripción minuciosa de ceremoniales que se celebran en la corte, como entradas reales o banquetes. Todo ello se presenta bajo un complejo ensamblaje alegórico-mitológico que tiene como finalidad constituirse, principalmente, como un panegírico de la corte de los $\mathrm{Re}$ yes Católicos, que se identifica con el Paraíso Terrenal.

Por su parte, Juana de Castilla fue la dedicataria de dos breves composiciones que se enmarcan en el contexto de la inesperada muerte del rey Felipe de Habsburgo, lo que nos permite establecer el año 1506 como fecha de composición. La primera es la que Dutton identifica en MP2 con A la Reyna Doña Juana nuestra señora quando murio el Rey Don phelipe nuestro señor (ID 1944). Es una copla real donde Pedro de Gracia Dei coloca a Juana de Castilla en primera persona, como transmisora de un mensaje cargado de tintes emocionales, un estilo que no encontramos en ninguna otra obra del autor. La reina Juana no expresa únicamente el dolor por la muerte de su marido, sino también por la pérdida de su madre, la reina Isabel - «yo perdí la madre mía»- y el desprecio que sufrió por parte de su padre -«de mi padre só olvidada». 
En esta línea y, también en MP2, se transmite un poema titulado $A$ la muerte del rey don Phelipe, que, aunque Dutton identifica como parte del Tratado de todos los reyes que en España ha habido desde los godos, parece, más bien, una composición independiente. No encaja con la línea temática de ID 1942 y, aunque se copia a continuación, lleva una rúbrica propia. Es una décima que recrea los rumores que giraron en torno al posible envenenamiento que pudo sufrir Felipe el Hermoso, aunque el autor concluye, finalmente, que tras «catar el cuerpo» no encontraron indicios de que fuese «muerto con yerbas».

Hasta aquí, damos cuenta de todas las obras que documenta Dutton a partir de los cuatro testimonios que recoge en El cancionero del siglo $X V$, así como de aquellos textos que se transmiten en otros manuscritos y que, con un alto grado de fiabilidad, podemos atribuir a Pedro de Gracia Dei: no solo porque consta en las rúbricas, sino porque tanto los temas como los distintos mecanismos poéticos que advertimos así lo respaldan.

\section{OBRAS ATRIBUIDAS}

Cabe señalar, en este punto, algunas composiciones que parte de la crítica ha considerado de nuestro autor. Infantes fue el primero en atribuir a Pedro de Gracia Dei una obra que intitula Tratados especiales de los linages de Leyba y de la Cueva ${ }^{17} \mathrm{y}$, aunque cuestiona su autoría, no ofrece ningún argumento al respecto. Que tengamos constancia, esos textos se transmiten únicamente en dos testimonios: el ms. 3564 de la BNE (ff. 19r-44r) y el ms. 69 de la Biblioteca Lambert Mata (ff. 75r-105r). Sin embargo, el nombre de Gracia Dei no aparece en ninguna parte, ni siquiera en el título, aspecto que resulta impropio en la transmisión de la poesía de Gracia Dei, ya que el resto de obras se caracteriza, precisamente, por la extensión de las rúbricas y los datos que presentan: no solo se deja clara la autoría de Gracia Dei, sino que, además, enumeran los distintos cargos que desempeñó dentro de la corte de los Reyes Católicos e, incluso, después, como criado de Juana de Castilla y Felipe de Habsburgo. De estas rúbricas se infiere, por tanto, un interés especial de los copistas por ofrecer todas las noticias posibles sobre el autor, así como el prestigio que tenía su figura, máxime si atendemos, además, a algunos manuscritos, donde se le denomina «sabio varón». Es extraño, por tanto, que no haya ninguna mención a Pedro de Gracia Dei a lo largo de estos tratados.

El autor, desconocido hasta ahora, toma distintas fuentes para la confección de sus textos, tanto en prosa como en verso, entre las que destacan obras de genealogistas y heraldistas reputados de los siglos XV y XVI, como Luis Zapata o Pedro de Gracia Dei, del que se incluye una estrofa que se corresponde con el linaje de Leiva y que forma parte de

\footnotetext{
${ }^{17}$ Víctor Infantes, art. cit., p. 46.
} 
los Blasones de las armas e insignias de los mejores y más principales linajes de Castilla (ID 1941). Debieron de ser estos versos que, además, aparecen al principio de la obra, lo que llevaría a Infantes a considerar de Pedro de Gracia Dei los tratados completos.

Se ha intentado demostrar que Pedro de Gracia Dei es el autor de las coplas iniciales y finales que forman parte del Libro de los pensamientos variables $^{18}$. La obra, de tradición mono-testimonial, se conserva en el ms. 6642 de la BNE. El juicio sobre la autoría se sostiene, principalmente, por el carácter laudatorio de los versos, dirigidos expresamente a Isabel la Católica: «Reina de muy gran grandeza / y en todas cosas gran reina...». Estas coplas se insertan, en efecto, en la misma línea de Las XV preguntas del papa Julio... o del proemio que antecede al Tratado de los reyes que en España ha habido desde los godos. Solo por cuestiones temáticas, Perea Rodríguez propone a Gracia Dei también como autor de las Coplas fechas a los altos estados de los reys nuestros señores (96*AE, ID 4693) y las Coplas fechas sobre el casamiento de la hija del rey de España $\left(96^{*} \mathrm{CH} \text {, ID } 4695\right)^{19}$. Ambos poemas recrean dos acontecimientos muy importantes que tuvieron cabida en el entorno regio: el matrimonio del príncipe Juan con Margarita de Austria y el enlace de Juana de Castilla con Felipe de Habsburgo.

El contexto de producción de las tres obras está vinculado directamente con los Reyes Católicos, de manera que, en principio, podrían responder a las tónicas habituales dentro de la obra poética de Pedro de Gracia Dei. No obstante, tal afirmación requeriría un profundo estudio literario partiendo, fundamentalmente, del usus escribendi del autor, análisis que desborda el objetivo de esta investigación.

Con este capítulo se han intentado aclarar todos los problemas que rodean la obra poética de Pedro de Gracia, que se manifiestan ya en $E l$ cancionero del siglo $\mathrm{XV}$, pues el mero hecho de tener que recurrir a la duplicación de un mismo ID o el uso de la referencia bis evidencia la confusión de Dutton. La revisión del corpus poético de Gracia Dei a partir de todos los testimonios localizados nos permite, por tanto, catalogar un total de quince obras, entre las que se incluyen, por ser proemios, las dos coplas introductorias de los poemas ID 1942 e ID 6966 ${ }^{\text {bis. }}$. De esos quince textos, trece se transmiten por vía manuscrita y se insertan, como hemos advertido, en complejos procesos de transmisión textual. Además, el grado de difusión es muy distinto entre unas composiciones

${ }^{18}$ Francisco López Estrada, «Anuncios renacentistas en el Libro de los pensamientos variables», en Homenaje a Eugenio Asensio, Madrid, Gredos, 1988, pp. 277-289; Óscar Perea Rodríguez, «La utopía política en la literatura castellana del siglo xv: el Libro de los pensamientos variables (BNM, ms. 6642)», eHumanista: Journal of Iberian Studies, 2 (2002), pp. 23-62; Esther Gómez Sierra (ed.), Diálogo entre el prudente rey y el sabio aldeano (olim Libro de los pensamientos variables), London, Departament of Hispanic Studies-Queen Mary and Westfield College, 2000.

${ }^{19}$ Óscar Perea Rodríguez, «“Alta reina esclarecida”», art. cit., p. 1373. n. 133 y pp. 1374-1375. 
y otras: por un lado, encontramos cuatro unica, con las limitaciones que ello conlleva para su estudio y edición crítica; por otro, disponemos de textos que se han transmitido en más de quince fuentes, lo que nos llevaría también a no pocas reflexiones ecdóticas. No obstante, la delimitación de este corpus intenta facilitar cualquier acercamiento a la obra en verso de Pedro de Gracia Dei.

\section{Apéndice. Catálogo de obras poéticas de Pedro de Gracia Dei}

El cómputo total de las obras poéticas de Pedro de Gracia Dei asciende a quince: catorce textos conservados y uno que conocemos a partir del Regestrum de Hernando Colón, más allá de otras cuatro composiciones que se le han atribuido, a partir de criterios históricos o literarios. Inician este catálogo las que no presentan dudas de atribución y se ordenan a partir de los IDs asignados por Dutton en El cancionero del siglo $\mathrm{XV}$. Cuando no tienen un ID asignado, así se indica y se catalogan a continuación del resto, alfabetizadas por su rúbrica. Tras estas, se indica la composición perdida y, después, las atribuidas.

La presencia de IDs con «bis» indica las dificultades que tuvo Dutton para delimitar la compleja textualidad y transmisión de los textos de Pedro de Gracia Dei. Aun respetando su numeración, se propone, en algún caso, un ID alternativo entre paréntesis, que puede contribuir a una mejor identificación de los textos. Este índice no se adapta, sensu stricto, al modelo convencional que presenta El cancionero del siglo $\mathrm{XV}$, pues de esta forma se perderían todas las diferencias que existen en los íncipit o éxplicit de una misma composición, así como también se duplicarían esquemas métricos. Por ello, las obras se agrupan a partir de dos aspectos coincidentes en el conjunto de la transmisión textual: íncipit y éxplicit, por un lado, y estructura métrica, por el otro. No se distingue, para el caso de primeros y últimos versos, variantes ortográficas o que no sean de significado. Para la identificación de fuentes, se mantienen las siglas de los cuatro testimonios documentados por Dutton; el resto se indica con la abreviatura del fondo bibliográfico en el que se conserva, seguida de su correspondiente signatura.

$\mathrm{BA}=$ Biblioteca da Ajuda, Lisboa

$\mathrm{BCS}=$ Biblioteca Capitular y Colombina, Sevilla

$\mathrm{BDR}=$ Biblioteca del Monasterio de las Descalzas Reales, Madrid

BLM= Biblioteca Lambert Mata, Ripoll

$\mathrm{BNE}=$ Biblioteca Nacional de España, Madrid

$\mathrm{BNP}=$ Biblioteca Nacional de Portugal, Lisboa

$\mathrm{RAH}=$ Real Academia de la Historia, Madrid

$\mathrm{RBE}=$ Real Biblioteca del Monasterio de El Escorial, Madrid

$\mathrm{RBP}=$ Real Biblioteca de Palacio, Madrid 


\section{1) ID $1941^{20}$}

Blasones de las armas e insignias de los mejores y más principales linajes de Castilla ${ }^{21}$

NH6 (ff. 34v-58va). Ínc.: «Con parte de las Reales / en purpura vi dos pieças»; éxpl.: «y hace esclavo del señor / al que le rendiera amigo»

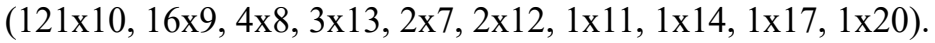

BCS, ms. 58-4-6 (ff. 42r-69v); BNE, ms. 7864 (ff. 15ra-22ra); BNP, COD. 1155 (ff. 47r-83r). Ínc.: «Con parte de las reales / en purpura vi dos piezas»; éxpl.: «don Manuel le llamo / legitimo de Castilla» (93x10, $3 \times 13,3 \times 9,2 \times 14,2 \times 8,2 \times 51 \times 11)$.

BLM, ms. 69 (ff. 31r-73r); BNE, ms. 18045 (ff. 28v-50r). Ínc.: «Con parte de las reales / en purpura vi dos pieças»; éxpl.: «varones del somenaje $[$ sic $]$ / de otro hecho y consejo» $(72 \times 10,3 \times 13,3 \times 9,2 \times 8,1 \times 14$, $1 \times 11)$.

BNE, mss. 3231 (ff. 35v-91v), 3769 (ff. 26v-64r), 5911 (ff. 5va18rb). Ínc.: «Con parte de las reales / en purpura vi dos piezas»; éxpl.: «y haze esclavo del señor / al que le rendiere amigo» (mismos íncipit y éxplicit que NH6, pero variación en la estructura métrica: 119x10, 19x9, $4 \times 8,3 \times 13,2 \times 7,2 \times 12,1 \times 11,1 \times 14,1 \times 17,1 \times 20$ ).

BNE, ms. 3449 (ff. 9ra-13vb). Ínc.: «Con parte de los reales / en purpura vi dos pieças»; éxpl.: «uizo [sic] castillos le dieron / segun quel Rei Iusticia» $(72 \times 10,2 \times 13,1 \times 8,4 \times 9,1 \times 11,2 \times 14)$.

BNE, ms. 3564 (ff. 45r-89v). Ínc.: «Es solosar [sic] en Viscaya / de quien llaman Esquibeles»; éxpl.: «sacando su Rey fuera / por do le dieron su paloma» $(49 \times 10,16 \times 8,2 \times 5)$.

BNE, ms. 6175 (ff. 262ra-269vb). Ínc.: «Azules y blancos veros / De Nureña y Visuezes»; éxpl.: «de dos en dos no rendilla / oro para ser nombrada» $(51 \times 10,16 \times 9,2 \times 8,2 \times 12,1 \times 7,1 \times 20)$.

${ }^{20}$ Algunas estrofas de esta obra se transmiten conjuntamente con coplas que forman parte de ID $6967^{\text {bis }}$, bajo el título Coplas de Gracia Dei en loor de algunos linajes y ciudades de Castilla: Biblioteca da Ajuda, ms. 50-I-41 (ff. 57-164). Ínc.: «Para blasonar a Castilla / es tan alta su sustancia»; éxpl.: «es su arco margaritado / de castillo nobles leales» $(81 \times 10,14 \times 9,4 \times 8$, $2 \times 12,2 \times 14,1 \times 4,1 \times 5,1 \times 6,1 \times 11,1 \times 13$ ). BNE, ms. 1367 (ff. 286ra-290rb). Ínc.: «De doce porque la fee / capitanes de la luz»; éxpl.: «magnanimo y liberal / gran celador de la ley» (57x10, 4x9, 1x5). BNE, ms. 12612 (ff. 28r-132v); RBP, ms. II/660 (ff. 23v-138v). Real Biblioteca de Palacio, MP2 (ff. 125va-132vb). Ínc.: «De doce que por la fee / capitanes de la luz»; éxpl.: «magnanimo y liveral / gran çelador de la ley» $(56 \times 10,4 \times 9,1 \times 5)$. No se trata de una obra distinta de Pedro de Gracia Dei, sino de una composición en que se aglutinan coplas de dos textos diferentes. Es por ello que no la consignamos como obra independiente, pues es resultado de un proceso de copia y no responde a la voluntad del autor.

${ }^{21}$ Esta es la rúbrica predominante en el conjunto de los testimonios. Sin embargo, en el ms. 1367 de la BNE, se transmite como Descendencia de algunos linages nobles de castilla; el ms. 3564 de la BNE no incluye rúbrica. En el caso del ms. 12612 de la BNE y del ms. II/660 de la RBP, se introducen varias coplas de ID 1941 con otras que pertenecen al Licenciado Molina y Damian de Goes, de manera que la rúbrica incluye a los tres autores. 


\section{2) ID 1942}

La descendencia de los Reyes de Castilla ${ }^{22}$

MP2 (ff. 132vb-135rb) ${ }^{23}$; NH6 (ff. 7r-11v); BA, ms. 50-I-41 (ff. 4-14); BCS, ms. 58-4-6 (ff. 4r-9r); BNE, mss. 3231 (ff. 1r-7r), 3449 (ff. 2ra-3rb), 3564 (ff. 1r-4r), 7864 (ff. 3ra-4vb); BNP, COD. 1155 (ff. 1r $7 \mathrm{v})$. Ínc.: «Año del omnipotente / de trescientos y cinquenta»; éxpl.: «y nuevas gentes hallando / a Napoles recobraron» $(19 \times 10)$.

BDR, ms. F/30 (ff. 147ra-147va); RAH, ms. 9/271 (ff. 267va-268ra), RBE, ms. h. II. 21 (ff. 112r-113r); RBP, ms. II/660 (ff. 139r-140r). Ínc.: «Pelayo fue restaurante / el osso mato a favila»; éxpl.: «don Hernando e Ysabel / los dos bien auenturados» (fragmento, 7x10) ${ }^{24}$.

BLM, ms. 69 (ff. 2r-6v); BNE, mss. 3564 (ff. 1r-4r), 18045 (ff. 1r$5 \mathrm{v})$. Ínc.: «Año del omnipotente / de trescientos y cinquenta»; éxpl.: «y nuevas gentes pallando [sic] / a Napoles recobraron» (19x10).

BNE, ms. 6175 (ff. 258ra-259vb). Ínc.: Año del omnipotente / de trescientos y cinquenta; éxpl.: D. ${ }^{\mathrm{n}}$ Fernando e Ysabel / los dos vienaventurados» $(18 \times 10)$.

\section{3) ID 1943}

\section{Las mujeres reynas en España ${ }^{25}$}

MN57 (f. 108r); MP2 (ff. 135rb-135va); BNE, mss. 1367 (f. 331rb), 3231 (f. 35r), 3769 (f. 25r), 5911 (f. 5va), 12612 (f. 74v); RBP, ms. II/660 (f. 73r). Ínc.: «Yberia nombre le dio / la loba sanctos exemplos»; éxpl.: «Ysabel en cada cosa / perfecion de todas ellas» $(1 \mathrm{x} 10)$.

NH6 (f. 34r); BNE, mss. 3449 (f. 8va), 3564 (f. 18r), 7864 (f. 12rb), 18045 (f. 28r); BCS, ms. 58-4-6 (f. 34r); BLM, ms. 69 (f. 30r); BNP, COD. 1155 (f. 38r); RBP, ms. II/660 (f. 143r). Ínc.: «Eugenia nombre le dio / la loba sanctos exemplos»; éxpl.: «Ysabel en cada cosa / perfecion de todas ellas» $(1 \mathrm{x} 10)$.

${ }^{22}$ Mantenemos el título que le asigna Dutton a partir de MP2, si bien en el ms. 69 de la BLM se transmite como Tratado de todos los reyes que en España ha habido desde los godos. También BETA o el IGM tienden a consignar este segundo título en las descripciones internas. No obstante, la mayoría de testimonios no incluye rúbrica, pues la obra comienza después de ID $6964^{\text {bis }}$, la copla que funciona como introducción a este poema. El ms. 1367 de la BNE intitula la composición Blasones de armas y linages de Gracia Dey, como si se tratase del texto ID 1941, de manera que se puede crear una confusión.

${ }^{23}$ Identificamos esta obra en MP2 a partir del modelo de 19 coplas, pues suponemos que se trata del esquema métrico completo. Sin embargo, en este cancionero se incluyen otras tres estrofas sin rúbrica que no forman parte de esta composición.

${ }^{24}$ En el ms. 18045 de la BNE (ff. 53va-54ra) se duplican también estas siete coplas. Este testimonio no está emparentado con las fuentes en que se transmite de forma fragmentaria, sino que se trata de un aspecto relacionado con el proceso de compilación del códice, pues el copista incluye coplas de Gracia Dei que pertenecen a varias obras diferentes.

${ }^{25}$ Este es el título que se repite con más frecuencia en el conjunto de la transmisión textual. No obstante, en algunos testimonios se transmite como Esparsa suya en que pone muchos nombres de reinas de españa (MP2 y RBE, ms. h. II. 21) o Reynas propietarias (MN57). 
BDR, ms. F/30 (f. 147va-vb); RAH, ms. 9/271 (f. 268ra); RBE, ms. h. II. 21 (f. 113r); Ínc.: «Ygeria nombre le dio / la loba sanctos exemplos»; éxpl.: «Ysabel en cada cosa / perfecion de todas ellas» (1x10).

\section{4) ID 1944}

A la Reyna Doña Juana nuestra señora cuando murió el rey Don phelipe nuestro señor.

MP2 (f. 135va). Ínc.: «Adonde se hallaria / quien fuese tan desdichado»; éxpl.: «y aora de entre las manos / a mi bien llevole dios» (1x10).

\section{5) ID 4692}

Criança y virtuosa dotrina.

$89 *$ GD (20 hojas) «La criança y virtuosa dotrina dedicada a la muy / illustre y esclarecida señora doña Isabel primera infante de Castilla...». Prohemio. Ínc.: «A vos Diana primera leona / a quien celio promete oceano»; éxpl.: «de los cien pares con los cinquenta / bien como niño sobre gigante» $(158 \times 8)$.

\section{6) ID $6964^{\text {bis }}$ I 1942}

Comiença y dirige la obra a la Reyna doña ysabel.

NH6 (f. 7r); BCS, ms. 58-4-6 (f. 3r); BNE, mss. 3231 (f. 1r), 3449 (f. 2ra), 7864 (f. 2v); BNP, COD. 1155 (f. IIr). Ínc.: «Muy alta muy poderosa / del mundo mayor señora»; éxpl.: «de los sçiticos abuelos / vos cuentan los Reyes ochenta» (1x10).

BA, ms. 50-I-41 (f. 4). Ínc.: «Mui alta e mui poderosa / del mundo maior señora»; éxpl.: «do sciticos aguelos / nos cuentan los reyes ochenta» $(1 \times 10)$.

BLM, ms. 69 (f. 2r); BNE, ms. 18045 (f. 1r). Ínc.: «Muy alta muy poderosa / del mundo mayor señora»; éxpl.: «de los sciticos Abuelos / os cuentan los Reies ochenta» (1x10).

BNE, ms. 3564 (f. 1r). Ínc.: «Muy alta muy poderosa / del mundo mior [sic] señora»; éxpl.: «de los sciticos Abuelos / os cuentan los Reies ochenta» $(1 \mathrm{x} 10)$.

\section{7) ID $6965^{\text {bis }}$ (quizá ID 6965 I $\left.6966^{\text {bis }}\right)^{26}$}

Genealogía y blasón de los reyes de Castilla ${ }^{27}$

NH6 (f. 12r); BA, ms. 50-I-41 (ff. 15-16); BCS, ms. 58-4-6 (f. 10r); BNE, mss. 3231 (f. 7r-v), 3449 (f. 3va), 7864 (f. 5ra-rb); BNP, COD.

${ }^{26}$ Aunque Dutton asigna a esta obra el ID $6965^{\text {bis }}$, cabe la posibilidad, a partir de su metodología, de identificar este texto con el ID 6965 I $6966^{\text {bis }}$, pues se trata de una estrofa que funciona siempre como introducción de ID $6966^{\text {bis. }}$.

${ }^{27}$ Recordemos que esta rúbrica encaja mejor con el poema completo (ID6966 ${ }^{\text {bis }}$ y no solo con la copla que funciona de introducción. 
1155 (f. 8r-v); Ínc.: «Quien los blasones leyere / de la real genealogia»; éxpl.: «y mediando cubre clausa / lo que en junta no se alaua» $(1 \mathrm{x} 10)$.

BLM, ms. 69 (f. 7r); BNE, mss. 3564 (f. 4r), 18045 (f. 6r). Ínc.: «Quien los blasones leyera / de la real genealogia»; éxpl.: «y mediando cubre clausa / lo que en junta no se alaua» $(1 \times 10)$.

\section{8) ID $6966^{\text {bis }}$ (quizá ID 6966) ${ }^{28}$}

De los Reyes godos.

NH6 (ff. 12r-33v); BCS, ms. 58-4-6 (ff. 10v-33r); BLM, ms. 69 (ff. 7r-29v); BNE, mss. 3449 (ff. 3va-8rb), 3564 (ff. 4r-18r), 7864 (ff. 5ra12rb), 18045 (ff. 6r-27v). Ínc.: «Dos leones colorados / de Atanarico animoso»; éxpl.: «quien dio tales alegrías / a sus reynos tan lozanos» $(86 \times 10,1 \times 9)$.

BA, ms. 50-I-41 (ff. 16-55). Ínc.: «Dos leones colorados / de athanarico animoso»; éxpl.: «hiço y pago quedo a quedo / a Salamanca las sciencias» (fragmento, 75x10).

BNE, ms. 3231 (ff. 7v-34v). Ínc.: «Dos leones colorados / de Atanarico animoso»; éxpl.: «quien dio tales alegrías / a sus reynos tan lozanos» $(80 \times 10,1 \times 9)$.

BNE, mss. 3769 (ff. 4r-21v), 5911 (ff. 1ra-5va). Ínc.: «Las leyes de fuero llamadas / que haze el rey sisnando»; éxpl.: «quien dio tales alegrias / a sus reynos tan lozanos» (fragmento, 55x10, 1x9).

BNP, ms. COD. 1155 (ff. 8v-37v). Ínc.: «Dos leones colorados / de Atanarico animoso»; éxpl.: «quien dio tales alegrías / a sus reynos tan lozanos» $(87 \times 10)$.

\section{9) ID $6967^{\text {bis }}$ (quizá ID 6967) ${ }^{29}$}

Blasones de Ciudades y Villas ${ }^{30}$

NH6 (ff. 69va-71vb). Ínc.: «Vi el carro triumphal / en la noble Carrion»; éxpl.: «que Castilla castellanos / Da Buelos Padres defendida» $(23 \times 10,2 \times 5,1 \times 7,1 \times 9,1 \times 11)$.

BCS, ms. 58-4-6 (ff. 35v-41r); BNE, ms. 7864 (ff. 13ra-14vb); BNP, COD. 1155 (ff. 39r-46v). Ínc.: «Para blasonar a Castilla / es tan alta su substancia»; éxpl.: «que la gran Roma del Tybre / nunca se mostro tan bella» $(22 \times 10,1 \times 9)$.

${ }^{28}$ La referencia «bis» no parece necesaria, pues Dutton identifica este texto a partir del cancionero NH6 y los límites de la composición quedan claros. Además, ninguna copla se utiliza como material poético para otra obra distinta, como sí ocurre en otros casos.

${ }^{29}$ Es probable que Dutton asigne la referencia «bis» a partir del texto que transmite MP2, donde aparecen conjuntamente estrofas que forman parte de ID $1941 \mathrm{y}$ de esta obra. No obstante, en NH6 y otros testimonios se transmite como una composición independiente.

${ }^{30}$ En el ms. 7864 de la BNE se transmite bajo el ambiguo título de Blasones, denominación que puede dar lugar a confusión, pues podría tratarse de los Blasones de ciudades y villas, como es el caso, pero también de los Blasones y armas de los mejores y más principales linajes de Castilla (ID 1941). 
BNE, mss. 3231 (ff. 92r-102v), 3769 (ff. 64v-72v), 5911 (ff. 18va21rb). Ínc.: «Vi el carro triumphal / en la noble Carrion»; éxpl.: «que Castilla castellanos / da buelos padres defendida» $(23 \times 10,2 \times 5,2 \times 9,1 \times 7)$.

BNE, ms. 3449 (f. 8va-vb). Ínc.: «Para blasonar a Castilla / es tan alta su substancia»; éxpl.: «y sus personas fiaron / de los fieles Tordesillas» $(3 \times 10,1 \times 9,1 \times 11)$.

BNE, ms. 6175 (ff. 259vb-262ra). Ínc.: «Vi el carro triunfal / en la noble Carrion»; éxpl.: «siempre fuiste atrebida / ardiol [sic] y muy torreada» $(16 \times 10,5 \times 9)$.

\section{0) ID 8324 V 1942}

Suma de todos los Reyes que a avido en españa desde el tiempo los godos.

MN57 (ff. 104v-107v). Ínc.: «Año del omnipotente / de tresçientos y cinquenta»; éxpl.: «que a cualquier Rey coronado / de los donde el desçendio» (19x10).

\section{1) SIN ID}

A la muerte del Rey don Philipe.

MP2 (f. 135ra). Ínc.: «Despues que muerto Al Rey vieron / al modo de Reyes godos»; éxpl.: «mas sospecha no hallaron, / que fuese muerto con yerbas» $(1 \mathrm{x} 10)$.

\section{2) SIN ID}

Casas y solares nobles de España ${ }^{31}$

BNE, mss. 3231 (ff. 109r-134r), 3769 (ff. 80r-110r), 5911 (ff. 23rb$32 \mathrm{vb}$ ). Ínc.: «De aqueste solar bien sano / fue yanguas dos cameros»; éxpl.: «con sus tiempos no noveles / matiz de sus blasones» (106x10, $2 \times 9,1 \times 6,1 \times 8,1 \times 11)$.

BNE, ms. 18053 (ff. 18v-122rb). Ínc.: «De aqueste solar bien sano / son yanguas y los cameros»; éxpl.: «los que los treze roeles / tiene tomados por guia» $(280 \times 10,2 \times 9,1 \times 6,1 \times 8,1 \times 11)$.

\section{3) SIN ID}

Coplas de los escudos de los príncipes del mundo ${ }^{32}$

BNE, mss. 3231 (ff. 104r-109r), 3769 (ff. 74r-80r), 5911 (ff. 21rb23rb), 18053, (ff. 1va-17ra). Ínc.: «La ciudad de Jerusalem / es la que

${ }^{31}$ Este es el título que consignan los dos grandes repertorios que manejamos: BETA y el IGM. Se trata de un título convencional para identificar el texto, pues en los manuscritos se transmite sin rúbrica, a continuación de las Coplas de los escudos de los príncipes del mundo, mediante la única indicación de «Comienzan los escudos en A. De las armas de los Arellanos». Solo el ms. 18053 de la BNE añade lo siguiente: «Comiençan las armas y blasones de los cavalleros y nobles despaña».

${ }^{32}$ Se copia sin rúbrica en el ms. 18380 de la BNE. 
primero fue»; éxpl.: «la cruz negra y oro escudo / con seis baculos dorados» $(23 \times 10,1 \times 5)$.

BNE, ms. 9087 (ff. 276r-305r). Ínc.: «La ciudad de Jerusalem / es la que primero fue»; éxpl.: «la cruz negra y oro escudo / con seis baculos dorados» $(21 \times 10,2 \times 9,1 \times 5)$.

BNE, ms. 18380 (ff. 1r-9v). Ínc.: «La ciudad de Jerusalem / es la que primero fue»; éxpl.: «de las nabas de Tolosa / de tanta morisma llenas»

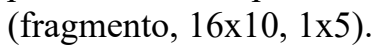

\section{4) SIN ID}

Las XV preguntas que hizo el papa Julio sobre las excelencias de la reina doña Isabel.

BNE, ms. 3346 (ff. 268r-269r); RBP, ms. II/660: ff. 140v-143r). Pregunta. Ínc.: «Ysabel quien llevo dios / quien era pregunto quien»; éxpl.: «y quanto vivio rennando / como ydo se sepulto». Respuesta. Quien era. Ínc.: «Muy alta muy poderosa / del mundo mayor señora»; éxpl.: «do yaze tierra con tierra / dios le de santa morada» $(12 \times 10)$.

OBRAS PERDIDAS

\section{5) SIN ID}

Exçelencias de la Reina doña Isabel de Castilla en coplas castellanas. «I. para poder entender. De. Ganeis a Ierusalen al principio estan las cosas de que trata es en $4^{\circ} .2$ col. costo en Valladolid $8 \mathrm{mrs}$. a 12. de noviembre. de 1524». Regestrum, $\mathrm{n}^{\circ} 4007$.

OBRAS ATRIBUIDAS ${ }^{33}$

\section{6) ID 4693}

Coplas fechas a los altos estados de los reys nuestros señores.

96*AE (4 hojas). Ínc.: «Alto dios omnipotente / resplandor de resplandores»; éxpl.: «un señor estableciste / sobre todos los mayores» (37x8).

\section{7) ID 4695}

Coplas fechas sobre el casamiento de la hija del rey despaña.

$96 * \mathrm{CH}$ (4 hojas). Ínc.: «Altos reyes poderosos / por manos de dios ungidos»; éxpl.: «en sus tierras gozaran / ende mas en el imperio» $(31 \times 10)^{34}$.

${ }^{33}$ Además de las cuatro composiciones poéticas que se indexan en este apartado, Infantes atribuye a Gracia Dei los Tratados especiales de los linages de Leyba y de la Cueva (art. cit., p. 46), una obra en prosa que incluye estrofas de distintos genealogistas de los siglos XV y XVI, como Luis Zapata o el Licenciado Molina. La primera copla que aparece en el texto pertenece a Gracia Dei y forma parte de ID 1941. Debieron de ser estos versos lo que llevaron a Infantes a considerar de nuestro autor los tratados completos.

${ }^{34}$ En la BNE se conserva un manuscrito del siglo XVIII que contiene las Coplas fechas a los altos estados de los reys nuestros señores y las Coplas fechas sobre el casamiento de la hija 


\section{8) ID 8725}

Libro de los pensamientos variables.

BNE, ms. 6642 (ff. 1r-2v). Ínc.: «Reyna de muy gran grandeza / y en todas cosas gran Reyna»; éxpl.: «mas haga fin mi trovar / donde comiença mi prosa» $(8 \times 10)$.

\section{9) ID 8726}

Libro de los pensamientos variables.

BNE, ms. 6642 (f. 20r-v). Ínc.: «Mas si por ventura son / en grossero estilo escritas»; éxpl.: «reçiba la voluntad / ques por obra descontada» $(3 \times 10)^{35}$.

Recibido: $2 / 05 / 2020$

Aceptado: 20/08/2020

del rey despaña (mss. 12935/6).

${ }^{35}$ Dutton considera anónimas estas coplas iniciales y finales del Libro de los pensamientos variables (Dutton, op. cit., p. 371). Por su parte, López Estrada. Véase el epígrafe de «Obras atribuidas». 
$\cos$

\author{
TRANSMISIÓN TEXTUAL Y CATÁLOGO \\ DE la obra PoÉtica de PEdRo de Gracia DeI
}

\begin{abstract}
RESUmen: La transmisión textual de la obra en verso de Pedro de Gracia Dei se extiende durante cuatro siglos y se caracteriza, fundamentalmente, por la abundante difusión a través de copias manuscritas. Su producción poética evidencia una importante heteregoneidad temática, pues los textos se insertan en líneas tan diferentes como la heráldica, la historiografía o los panegíricos a Isabel la Católica. En este trabajo se establece un estudio y delimitación del corpus poético de Pedro de Gracia Dei a partir de los testimonios en que transmiten cada una de las composiciones, con el objetivo de solventar los problemas que afectan a los títulos, contenido y estructura métrica de los poemas.
\end{abstract}

Palabras Clave: Pedro de Gracia Dei, poesía de cancionero, codicología, transmisión textual, catálogo.

\title{
TEXTUAL TRANSMISSION AND CATALOGUE
} of POETIC WORK OF PEDro de Gracia DeI

ABSTRACT: The textual transmission of the work in verse by Pedro de Gracia Dei extends over four centuries and is characterized, fundamentally, by the abundant diffusion through copies manuscripts. His poetic production shows an important thematic heterogeneity, since the texts are inserted in lines as different as heraldry, historiography or eulogies to Isabel la Católica. This work establishes a study and delimitation of Pedro de Gracia Dei's poetic corpus from the testimonies in which each of the compositions is transmitted, with the aim of solving the problems that affect the titles, content and metric structure of the poems.

Keywords: Pedro de Gracia Dei, cancionero poetry, codicology, textual transmission, catalogue. 